\title{
Nash Consistent Representation of Constitutions: A Reaction to the Gibbard Paradox
}

Citation for published version (APA):

Peleg, B., Peters, H. J. M., \& Storcken, A. J. A. (2002). Nash Consistent Representation of Constitutions: A Reaction to the Gibbard Paradox. Mathematical Social Sciences, 43, 267-287. https://doi.org/10.1016/S0165-4896(01)00095-6

Document status and date:

Published: 01/01/2002

DOI:

10.1016/S0165-4896(01)00095-6

Document Version:

Publisher's PDF, also known as Version of record

\section{Please check the document version of this publication:}

- A submitted manuscript is the version of the article upon submission and before peer-review. There can be important differences between the submitted version and the official published version of record.

People interested in the research are advised to contact the author for the final version of the publication, or visit the DOI to the publisher's website.

- The final author version and the galley proof are versions of the publication after peer review.

- The final published version features the final layout of the paper including the volume, issue and page numbers.

Link to publication

\footnotetext{
General rights rights.

- You may freely distribute the URL identifying the publication in the public portal. please follow below link for the End User Agreement:

www.umlib.nl/taverne-license

Take down policy

If you believe that this document breaches copyright please contact us at:

repository@maastrichtuniversity.nl

providing details and we will investigate your claim.
}

Copyright and moral rights for the publications made accessible in the public portal are retained by the authors and/or other copyright owners and it is a condition of accessing publications that users recognise and abide by the legal requirements associated with these

- Users may download and print one copy of any publication from the public portal for the purpose of private study or research.

- You may not further distribute the material or use it for any profit-making activity or commercial gain

If the publication is distributed under the terms of Article $25 \mathrm{fa}$ of the Dutch Copyright Act, indicated by the "Taverne" license above, 


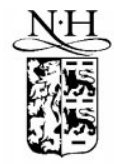

ELSEVIER
Mathematical Social Sciences 43 (2002) 267-287 mathematical

social

sciences

www.elsevier.com/locate/econbase

\title{
Nash consistent representation of constitutions: a reaction to the Gibbard paradox
}

\author{
Bezalel Peleg ${ }^{\mathrm{a}}$, Hans Peters ${ }^{\mathrm{b}, *}$, Ton Storcken ${ }^{\mathrm{b}}$ \\ a Department of Mathematics and Center for Rationality and Interactive Decision Theory, \\ The Hebrew University of Jerusalem, Feldman Building, Givat-Ram, 91904 Jerusalem, Israel \\ ${ }^{\mathrm{b}}$ University of Maastricht, Department of Quantitative Economics, P.O. Box 616, 6200 MD Maastricht, \\ The Netherlands
}

Received 1 August 2001; received in revised form 1 October 2001; accepted 31 October 2001

\begin{abstract}
The concept of an effectivity function is adopted as a formal model of a constitution. A game form models the actions available and permissible to individuals in a society. As a representation of the constitution such a game form should endow each group in society with the same power as it has under the constitution. Another desirable property is Nash consistency of the game form: whatever the individual preferences, the resulting game should be minimally stable in the sense of possessing a Nash equilibrium. A first main result of the paper is a characterization of all effectivity functions that have a Nash consistent representation for the case without special structure on the set of alternatives (social states). Next, a similar result is derived for the case where the set of alternatives is a topological space and the effectivity function is topological. As a special case, veto functions are considered. Further results concern Pareto optimality of Nash equilibrium outcomes. (C) 2002 Elsevier Science B.V. All rights reserved.
\end{abstract}

Keywords: Game form; Effectivity function; Nash consistency; Constitution

JEL classification: $\mathrm{O} 25 ; \mathrm{O} 26$

\section{Introduction}

The fascinating debate on the formalization of rights and constitutions which has taken place over the past decades shows that at least three constituents are inextricably

\footnotetext{
*Corresponding author. Tel.: +31-43-388-3288; fax: +31-43-388-4874.

E-mail addresses: pelegba@math.huji.ac.il (B. Peleg), h.peters@ke.unimaas.nl (H. Peters), t.storcken@ke.unimaas.nl (T. Storcken).
} 
bound up with these concepts: rights and constitutions by themselves; preferences of society members; and choices that society members can make.

Arrow (1967) defines a constitution as a 'well-behaved' social welfare function. According to this definition, a constitution is viewed as a social decision process strongly connected with individual preferences. As Arrow (1967, in particular p. 231) indicates, a difficulty arising from this definition is that social decision processes are subject to Arrow's impossibility theorem in social choice. Sen (1970, and many subsequent papers) adopts the idea of a 'recognized personal sphere' and requires that for every individual in society there should be at least one couple of social states over which that individual should be decisive. This individual right is imposed as a condition on a social choice function and, in this respect, Sen's approach has with Arrow's in common that it is based on individual preferences. Sen's 'impossibility of a Paretian liberal', which shows that individual rights are in conflict with Pareto optimality, has triggered a stream of literature discussing this genuine paradox. One of the focal points of this discussion is the distinction that can be made between individual rights - choices that the individual should have, and choices that the individual actually has-actions that he can take. In the well-known blue and white shirt example (in a somewhat different form proposed by Gibbard, 1974) each of two individuals can choose to wear either a white or a blue shirt. Sen's individual right condition would require an individual to be decisive, e.g. over the two social states in which the other individual wears blue, but this does not concur with the actual choices that are available and that, intuitively, constitute individual rights as well. For a detailed discussion of this so-called Gibbard paradox, see Gaertner et al. (1992).

Following Sugden (1985), Gaertner et al. (1992) proposed the use of game forms in order to formalize the idea of rights. In a game form, each individual chooses from a set of strategies. These strategies are the available as well as permissible actions and correspond to the rights of the individuals. An outcome function then chooses a social state for each combination of strategies. By adding the individual preferences, a game is obtained. In this approach there is a clear distinction between rights and preferences, but less so between rights and available choices. The right not to be killed could be modeled by not allowing murder as an available strategy for any of the individuals, but things are not always that simple. How about an individual's right to a minimum subsistence level? Trying to avoid such questions already seems a sufficient reason to further distinguish between rights and choices as well.

In the present paper we will follow the approach initiated by Gärdenfors (1981) and assume that rights are formalized by an effectivity function. Such a function describes for every group in society the sets of social states to which this group is entitled by its own rights. An effectivity function can be formulated independently of preferences and of choices. We will take it here as a primitive; for a possible foundation on an underlying constitution, see Peleg (1998). A society cannot function solely on the basis of a constitution, but additionally needs a collection of rules that delimit the actions available and permissible to individuals. For instance, freedom of speech is a basic right in most constitutions, but in practice one needs a set of rules that distinguish between an individual's right to express his own opinion on the one hand, and slander or discrimination on the other hand. Such rules are the legal means to reach social states 
that agree with the constitution. As another example, the right to a minimum subsistence level may be part of the constitution, but stealing is usually not regarded as a legal way to reach it. Similarly, we will search for a game form that 'represents' the effectivity function (constitution). The idea of representation will be given a precise meaning: the game form should endow each individual or group in the society with the same possibilities as intended by the original constitution. Viewing the game form as a decentralization of the constitution, no one should lose or gain power. But this is still not sufficient. Given that in a game form individuals choose their strategies independently, there is no guarantee that this will lead to a society that is stable, in the sense that no one would like to change his strategy. Therefore, we will impose the minimum requirement of Nash consistency: whatever the individual preferences, the resulting game should have at least one Nash equilibrium. The objective, then, of the present paper is to find necessary and sufficient conditions on an effectivity function to have a Nash consistent representation.

In more detail, the contents of the paper are as follows. After introducing definitions and notations in Section 2, in Section 3 we derive necessary and sufficient conditions for Nash consistent representation for the case in which there is no special structure imposed on the set of alternatives (i.e. social states). In Section 4 the set of alternatives is a topological space, and Nash consistent representation of topological effectivity functions is characterized. In Section 5 these results are applied to the special case of veto functions. Section 6 concludes. In Appendix A of the paper we present the construction of the game form that underlies all our representation results. This game form is of independent interest. It is maximal in the sense that, for any preferences, it allows for at least as many Nash equilibrium outcomes as any other representing game form. A surprising consequence of this will turn out to be that there is always a Pareto optimal Nash equilibrium outcome.

Apart from the references mentioned, other related papers include the following. For the unstructured case, Gurvich $(1976,1989)$ characterizes Nash consistent game forms for two individuals; see also Abdou (1995a,b). One of the early papers on topological effectivity functions is Keiding (1986). Our results on topological effectivity functions are motivated, in particular, by Abdou (1988). The much stronger condition of acceptability of a game form, implying not only Nash consistency but also Pareto optimality of any Nash equilibrium outcome, is studied by Hurwicz and Schmeidler (1978) and Dutta (1984). Deb et al. (1997) study the relation between Pareto optimality and other notions of equilibrium in game forms modeling rights. Implementation of a constitution is studied in Peleg and Winter (2000).

\section{Definitions and notations}

Throughout this paper, $A$ denotes the set of alternatives. The set $A$ may be finite or infinite; however, if $A$ is finite, then $|A| \geq 2$. (If $D$ is a finite set, then $|D|$ is the number of members of $D$.) A preference ordering on $A$ is a complete and transitive binary relation. The set of all preference orderings on $A$ is denoted by $W=W(A)$. If $R \in W$ and $x, y \in A$, then we write $x P y$ if $x R y$ and not $y R x$. If $a \in A$ and $R \in W$, then $L(a$, 
$R)=\{b \in A \mid a R b\}$ is the lower contour set of $a$, i.e. the set of alternatives not strictly preferred to $a$. For a set $S, W^{S}=\{f \mid f: S \rightarrow W\}$ is the set of mappings from $S$ to $W$.

For a set $D$, we denote by $P(D)$ the set of all subsets of $D$, and by $P_{0}(D)$ the set of all nonempty subsets of $D$. A structure on the set $A$ of alternatives is a subset $\mathscr{T}$ of $P_{0}(A)$ that satisfies: (i) $A \in \mathscr{T}$; (ii) $\{a\} \in \mathscr{T}$ for all $a \in A$; and (iii) if $B_{1}, B_{2} \in \mathscr{T}$ and $B_{1} \cap B_{2} \neq \emptyset$, then $B_{1} \cap B_{2} \in \mathscr{T}$. Special structures that will be considered in this paper are: the set of all nonempty subsets of $A$; and the set of all nonempty closed subsets of $A$, where $A$ is a topological space. The basic representation result Theorem A.1 in Appendix A holds for any structure.

Let $N=\{1, \ldots, n\}$ be the set of players, and let $\mathscr{T}$ be a structure on the set of alternatives $A$. An effectivity function (EF) is a function $E: P(N) \rightarrow P(\mathscr{T})$ that satisfies the following conditions: (i) $E(N)=\mathscr{T}$; (ii) $E(\emptyset)=\emptyset$; and (iii) $A \in E(S)$ for all $S \in P_{0}(N)$. As a general interpretation, $B \in E(S)$ means that coalition $S$ can force the final alternative to be an element of $B$. The interpretation of the three conditions is fairly obvious.

An effectivity function $E$ is superadditive if it satisfies the following condition: if $S_{i} \in P_{0}(N)$ and $B_{i} \in E\left(S_{i}\right)$ for $i=1,2$, and $S_{1} \cap S_{2}=\emptyset$, then $B_{1} \cap B_{2} \in E\left(S_{1} \cup S_{2}\right)$. The effectivity function $E$ is monotonic if

$$
\left[B \in E(S), B^{*} \in \mathscr{T}, B \subseteq B^{*} \text {, and } S \subseteq S^{*}\right] \Rightarrow B^{*} \in E\left(S^{*}\right) .
$$

Monotonicity and superadditivity of effectivity functions are natural properties in view of the interpretation given above. Moreover, effectivity functions derived from game forms (see below) have these properties.

The polar of $E$ is the effectivity function $E^{*}$ defined by: $E^{*}(\emptyset)=\emptyset$, and for $S \in P_{0}(N)$

$$
E^{*}(S)=\left\{B \in \mathscr{T} \mid B \cap B^{\prime} \neq \emptyset \text { for all } B^{\prime} \in E(N \backslash S)\right\} .
$$

Thus, if $B \in E^{*}(S)$, then the complementary coalition $N \backslash S$ cannot guarantee that the final alternative is not in $B . E$ is maximal if $E$ is superadditive and $E=E^{*}$.

Let $\hat{E}: P(N) \rightarrow P(\mathscr{T})$ be a function (not necessarily an effectivity function) that satisfies $E(\emptyset)=\emptyset$. The core of $\hat{E}$ with respect to $R^{N} \in W^{N}$ is defined in the following way. Let $B \in \mathscr{T}, S \in P_{0}(N)$, and $x \in A \backslash B$. We say that $B$ dominates $x$ via $S$ at $R^{N}$ if $B \in \hat{E}(S)$ and $b P^{i} x$ for all $b \in B$ and $i \in S$. Also, $x \in A$ is dominated at $R^{N}$ if there exist $B \in \mathscr{T}$ and $S \in P_{0}(N)$ such that $B$ dominates $x$ via $S$ at $R^{N}$. The core of $\hat{E}$ with respect to $R^{N}, C\left(\hat{E}, R^{N}\right)$, is the set of all undominated alternatives at $R^{N}$. For $Q \subseteq W$, we say that $\hat{E}$ is stable on $Q^{N}$ if $C\left(\hat{E}, R^{N}\right) \neq \emptyset$ for all $R^{N} \in Q^{N}$. For later reference, we also introduce the Pareto optimal set $P O\left(\hat{E}, R^{N}\right)$, which consists of all $x \in A$ that are not dominated via $N$.

We now turn to define some basic properties of game forms. A game form (GF) is an $(n+2)$-tuple $\Gamma=\left(\Sigma^{1}, \ldots, \Sigma^{n} ; \pi ; A\right)$, where (i) $\Sigma^{i}$ is the (non-empty) set of strategies of player $i \in N$; and (ii) $\pi: \Sigma^{1} \times \cdots \times \Sigma^{n} \rightarrow A$ is the outcome function. For $S \in$ $P_{0}(N)$ we denote $\Sigma^{S}=\times_{i \in S} \Sigma^{i}$. Also, we denote $\Sigma=\Sigma^{N}$. Let $R^{N} \in W^{N}$. The pair $(\Gamma$, $R^{N}$ ) defines, in an obvious way, a game in strategic form. A strategy combination $\sigma \in \Sigma$ is a Nash equilibrium $(\mathrm{NE})$ of $\left(\Gamma, R^{N}\right)$ if

$$
\left[\tau^{i} \in \Sigma^{i}, i \in N\right] \Rightarrow \pi(\sigma) R^{i} \pi\left(\sigma^{N \backslash\{i\}}, \tau^{i}\right) .
$$


(Here $\sigma^{N \backslash\{i\}}$ is the restriction of $\sigma$ to $N \backslash\{i\}$.) The set of all Nash equilibria of $\left(\Gamma, R^{N}\right)$ is denoted by $N E\left(\Gamma, R^{N}\right)$. For $Q \subseteq W, \Gamma$ is Nash consistent on $Q^{N}$ if $N E\left(\Gamma, R^{N}\right) \neq \emptyset$ for every $R^{N} \in Q^{N}$.

Let $\Gamma=\left(\Sigma^{1}, \ldots, \Sigma^{n} ; \pi ; A\right)$ be a game form and assume that $\pi$ is surjective. The effectivity function $E^{\Gamma}$, associated with $\Gamma$, is defined in the following way. For $S \in P_{0}(N)$ and $B \in P_{0}(A), S$ is effective for $B$ if there exists $\sigma^{S} \in \Sigma^{S}$ such that $\pi\left(\sigma^{S}\right.$,



$$
E^{\Gamma}(S)=\left\{B \in P_{0}(A) \mid S \text { is effective for } B\right\}, \text { for } S \in P_{0}(N) .
$$

( $E^{\Gamma}$ is defined in Moulin and Peleg (1982) where it is called the $\alpha$-EF of $\Gamma$.) Clearly, $E^{\Gamma}$ is superadditive and monotonic. Let $\mathscr{T}$ be a structure on $A$ and let $E: P(N) \rightarrow P(\mathscr{T})$ be an effectivity function. A game form $\Gamma$ is a representation of $E$ if $E(S)=E^{\Gamma}(S) \cap \mathscr{T}$ for every $S \in P_{0}(N)$. Basically, this means that the game form distributes the same power among the players as the effectivity function does.

Notational convention: instead of $E(\{i\})$ and $E^{*}(\{i\})$ we usually write $E(i)$ and $E^{*}(i)$.

\section{Existence of Nash consistent representations: the unstructured case}

In this section we provide necessary and sufficient conditions for the existence of a Nash consistent representation of an effectivity function for the case where there is no special structure on the set of alternatives $A$, i.e. $\mathscr{T}=P_{0}(A)$. An example is the classical model of social choice where $A$ is finite. See also Examples 3.9 and 3.10 .

Let $E: P(N) \rightarrow P\left(P_{0}(A)\right)$ be a superadditive and monotonic effectivity function. We associate with $E$ a function $\hat{E}: P(N) \rightarrow P\left(P_{0}(A)\right)$ in the following way:

$$
\hat{E}(S)= \begin{cases}E^{*}(i), & \text { if } S=\{i\}, i \in N \\ \{A\}, & \text { if }|S|>1, S \subseteq N \\ \emptyset, & \text { if } S=\emptyset\end{cases}
$$

The function $\hat{E}$ is called the residual of $E$. The importance of this function can be seen from the following lemma. Observe that, by Theorem A.1 in Appendix A, $E$ has a Nash consistent representation if, and only if, $E(N \backslash\{i\})$ is in a specific sense 'large' for every $i \in N$. Consequently, the polar $E^{*}(i)$ should be 'small', which allows $\hat{E}$ to have a nonempty core for every preference profile. The following lemma makes these intuitions precise.

Lemma 3.1. E has a Nash consistent representation if, and only if, $\hat{E}$ is stable.

Proof. Necessity: let $\Gamma=\left(\Sigma^{1}, \ldots, \Sigma^{n} ; \pi ; A\right)$ be an NC-representation of $E$, let $R^{N} \in W^{N}$, let $\sigma$ be an NE of $\left(\Gamma, R^{N}\right)$, and let $x=\pi(\sigma)$. As $\sigma$ is an NE,

$$
L\left(x, R^{i}\right) \in E^{\Gamma}(N \backslash\{i\})=E(N \backslash\{i\}) \text { for every } i \in N .
$$

Hence, $A L\left(x, R^{i}\right) \notin E^{*}(i)$ for all $i \in N$. Thus, $x \in C\left(\hat{E}, R^{N}\right)$. 
Sufficiency: assume that $\hat{E}$ is stable. Let $R^{N} \in W^{N}$ and $x \in C\left(\hat{E}, R^{N}\right)$. Then $A L(x$, $\left.R^{i}\right) \notin E^{*}(i)$ for every $i \in N$. Thus, by definition of $E^{*}$ and monotonicity, $L\left(x, R^{i}\right) \in$ $E(N \backslash\{i\})$ for every $i \in N$. Hence, by Theorem A.1, $E$ has an NC-representation.

We mention that for finite $A$ Lemma 3.1 is not entirely new. Danilov and Sotskov (1991, Chapter 2, Section 2) show that if $\Gamma$ is a Nash consistent game form then for every profile of preference orderings the set of individually rational alternatives in the associated polar effectivity function is nonempty: this is the only-if implication in Lemma 3.1. The if-implication can be derived from the proof of their Theorem 2.

Note that the necessity part of the proof of Lemma 3.1 implies that all Nash equilibrium outcomes of the game $\left(\Gamma, R^{N}\right)$ are in fact core elements of $\left(\hat{E}, R^{N}\right)$. By going over the proof of Theorem A.1 it can be checked that for the particular game form $\Gamma_{0}$ constructed in that proof, any core element is also a Nash equilibrium outcome. These facts are summarized in the following corollary.

Corollary 3.2. Let $E: P(N) \rightarrow P\left(P_{0}(A)\right)$ be an effectivity function. Then

(i) if $E$ has a Nash consistent representation $\Gamma$ with outcome function $\pi$, then $\pi(N E(\Gamma$, $\left.\left.R^{N}\right)\right) \subseteq C\left(\hat{E}, R^{N}\right)$ for all $R^{N} \in W^{N}$

(ii) if $E$ is superadditive and monotonic, and $C\left(\hat{E}, R^{N}\right) \neq \emptyset$ for all $R^{N} \in W^{N}$, then $\pi\left(N E\left(\Gamma_{0}, R^{N}\right)\right)=C\left(\hat{E}, R^{N}\right)$ for all $R^{N} \in W^{N}$, where $\pi$ is the outcome function of $\Gamma_{0}$.

This corollary implies in particular that the game form used in Appendix A admits the maximum number of Nash equilibrium outcomes. The following example shows that the inclusion in (i) of Corollary 3.2 may be strict.

Example 3.3. Let $N=\{1,2,3\}, A=\{a, b\}$, and consider the 'king maker' game form (Hurwicz and Schmeidler, 1978) $\Gamma=(\{2,3\},\{a, b\},\{a, b\} ; \pi ; A)$, where $\pi(2, x, y)=x$ and $\pi(3, x, y)=y$ for all $x, y \in A$. Then, for $E=E^{\Gamma}, E(S)=P_{0}(A)$ for all $S \subseteq N$ with $|S|>1$. Hence $E^{*}(i)=\{A\}$ for every $i \in N$. This implies $\hat{E}(S)=\{A\}$ for all $S \in P_{0}(N)$, so that $C\left(\hat{E}, R^{N}\right)=A$ for all $R^{N} \in W^{N}$. On the other hand, $\pi\left(N E\left(\Gamma, R^{N}\right)\right) \subseteq P O\left(R^{N}\right)$, which can be a strict subset of $A$.

We will next provide a combinatorial characterization of the stability of $\hat{E}$. Firstly, we enlarge each of the sets $E^{*}(i)$ by adding the intersection of every decreasing collection of sets in $E^{*}(i)$. The enlarged sets will be denoted by $I\left(E^{*}(i)\right), i \in N$.

Definition 3.4. Let $i \in N$ and $B \in P_{0}(A)$. Then $B \in I\left(E^{*}(i)\right)$ if there exists a (linearly pre-)ordered set $(T, \succeq)$ and a family of sets $B_{\tau} \in E^{*}(i), \tau \in T$, such that

(i) if $\theta, \tau \in T$ and $\tau \succeq \theta$, then $B_{\tau} \subseteq B_{\theta}$;

(ii) $B=\bigcap\left\{B_{\tau} \mid \tau \in T\right\}$. 
Note that if the set $A$ is finite, then $E^{*}(i)$ is not only a subset but actually equal to $I\left(E^{*}(i)\right)$ for every $i \in N$.

The announced characterization of the stability of $\hat{E}$ is the following.

Lemma 3.5. $\hat{E}$ is stable if, and only if,

$$
\left[B^{i} \in I\left(E^{*}(i)\right) \text { for all } i \in N\right] \Rightarrow \bigcap_{i=1}^{n} B^{i} \neq \emptyset \text {. }
$$

Proof. Sufficiency: assume that (1) is satisfied and let $R^{N} \in W^{N}$. For $i \in N$ let

$$
T^{i}=\left\{x \in A \mid A \backslash L\left(x, R^{i}\right) \in E^{*}(i)\right\},
$$

and order $T^{i}$ by $R^{i}$. For $x \in T^{i}$ let $B_{x}^{i}=A L\left(x, R^{i}\right)$. Then

$$
B^{i}=\bigcap\left\{B_{x}^{i} \mid x \in T^{i}\right\} \in I\left(E^{*}(i)\right) \text {. }
$$

By (1), $\bigcap_{i \in N} B^{i} \neq \emptyset$. Clearly, $C\left(\hat{E}, R^{N}\right) \supseteq \bigcap_{i \in N} B^{i}$.

Necessity: suppose that there exist $B^{i} \in I\left(E^{*}(i)\right), i \in N$, such that $\bigcap_{i \in N} B^{i}=\emptyset$. For every $i \in N$ there exists an ordered set $\left(T^{i}, \succeq^{i}\right)$ and a decreasing collection $B_{\tau}^{i} \in E^{*}(i)$, $\tau \in T^{i}$, such that $B^{i}=\bigcap\left\{B_{\tau}^{i} \mid \tau \in T^{i}\right\}$. Define $R^{i}$ on $A$ by

$$
x R^{i} y \Leftrightarrow\left[\text { for all } \tau \in T^{i}: y \in B_{\tau}^{i} \Rightarrow x \in B_{\tau}^{i}\right] .
$$

Then $x \in A$ is not dominated via $i$ at $R^{N}$ if and only if $x \in B^{i}$. Thus, $C\left(\hat{E}, R^{N}\right)$ $\subseteq \bigcap_{i \in N} B^{i}=\emptyset$, which completes the proof of the necessity part.

Lemmas 3.1 and 3.5 now yield the following theorem.

Theorem 3.6. Let $E: P(N) \rightarrow P\left(P_{0}(A)\right)$ be a superadditive and monotonic effectivity function. Then E has a Nash consistent representation if, and only if, (1) is satisfied.

As mentioned before, if the set of alternatives is finite, then $I\left(E^{*}(i)\right)=E^{*}(i)$ for every $i \in N$. So in that case condition (1) can be simplified. This results in the following corollary of Theorem 3.6.

Corollary 3.7. Let $E: P(N) \rightarrow P\left(P_{0}(A)\right)$ be a superadditive and monotonic effectivity function. If $|A|<\infty$, then $E$ has a Nash consistent representation if, and only if,

$$
\left[B^{i} \in E^{*}(i) \quad \text { for all } i \in N\right] \Rightarrow \bigcap_{i=1}^{n} B^{i} \neq \emptyset .
$$

Remark 3.8. If the effectivity function $E$ in Corollary 3.7 has a Nash consistent representation, then the representing game form can be constructed in such a way that it admits a Pareto optimal Nash equilibrium for every profile of preferences. See the end of Section 4 for details. 
The following two examples are direct applications of Corollary 3.7. Both examples were originally proposed by Gibbard (1974).

Example 3.9. Two individuals each have a white $(w)$ and a blue $(b)$ shirt (in Gibbard (1974) the colors refer to wallpaper). Each individual has the right to choose the color of his own shirt. Thus, $N=\{1,2\}$, and the set of alternatives (social states) is

$$
A=\{(w, w),(w, b),(b, w),(b, b)\},
$$

where for each alternative the first [second] letter refers to the color of the shirt of individual 1 [2]. For the associated effectivity function $E$ we have (cf. also Peleg, 1998): $E(1)$ contains all supersets of $\{(w, w),(w, b)\}$ and $\{(b, w),(b, b)\} ; E(2)$ contains all supersets of $\{(w, w),(b, w)\}$ and $\{(w, b),(b, b)\}$; and $E(N)=P_{0}(A)$. For the polar $E^{*}$ we have: $E^{*}(1)$ contains all supersets of

$$
\begin{array}{ll}
\{(w, w),(b, b)\}, & \{(w, w),(w, b)\}, \\
\{(b, w),(b, b)\}, & \{(b, w),(w, b)\},
\end{array}
$$

and $E^{*}(2)$ contains all supersets of

$$
\begin{array}{ll}
\{(w, w),(b, w)\}, & \{(w, w),(b, b)\}, \\
\{(w, b),(b, w)\}, & \{(w, b),(b, b)\} .
\end{array}
$$

Clearly, $E$ is superadditive and monotonic. Corollary 3.7 implies that it has a Nash consistent representation if, and only if, the intersection of any set in $E^{*}(1)$ with any set in $E^{*}(2)$ is nonempty. Obviously, this condition is not satisfied here. It follows, in particular, that the game form in which each individual chooses his own shirt, does not always have a Nash equilibrium. In one version of the so-called Gibbard paradox, player 1 is first of all a conformist, and for the rest prefers white to blue; player 2 also prefers white to blue but is first of all a nonconformist. This results in the bimatrix game

$$
w\left(\begin{array}{ll}
w & b \\
4,2 & 2,3 \\
1,4 & 3,1
\end{array}\right)
$$

which does not have a Nash equilibrium.

Example 3.10. In this example there are three players: Angelina (A), Edwin (E), and the male Judge (J). The set of alternatives is $A=\{s, e, j\}$, where $s$ means that Angelina stays single; $e$ that she marries Edwin; and $j$ that she marries the Judge. Each individual has the right to stay single, and Angelina and Edwin as well as Angelina and the Judge have the right to marry. For the associated effectivity function (cf. Peleg, 1998) we have: $E(\{\mathrm{~A}, \mathrm{E}\})$ contains all supersets of $\{s\}$ and $\{e\} ; E(\{\mathrm{~A}, \mathrm{~J}\})$ contains all supersets of $\{s\}$ and $\{j\} ; E(\{\mathrm{E}, \mathrm{J}\})$ contains all supersets of $\{s\} ; E(\mathrm{~A})$ contains all supersets of $\{s\} ; E(\mathrm{E})$ contains all supersets of $\{s, j\} ; E(\mathrm{~J})$ contains all supersets of $\{s, e\}$; and $E(\{\mathrm{~A}, \mathrm{E}$, $\mathrm{J}\})=P_{0}(A)$. Clearly, $E$ is superadditive and monotonic. For the polar $E^{*}$ we have: $E^{*}(\mathrm{~A})$ contains all subsets of $A$ that contain $s ; E^{*}(\mathrm{E})$ contains all subsets of $A$ that contain $s$ and $j$; and $E^{*}(\mathrm{~J})$ contains all subsets of $A$ that contain $s$ and $e$. Any triple of elements 
from $E^{*}(\mathrm{~A}), E^{*}(\mathrm{E})$, and $E^{*}(\mathrm{~J})$ has at least the alternative $s$ in common. Thus, for this effectivity function Corollary 3.7 implies the existence of a Nash consistent representation.

Remark 3.11. Condition (2) is equivalent to

$$
\left[B^{i} \notin E(N \backslash\{i\}) \text { for all } i \in N\right] \Rightarrow A \backslash \bigcup_{i=1}^{n} B^{i} \neq \emptyset .
$$

This condition is expressed in terms of the original effectivity function $E$.

The following example shows that (1) cannot be replaced by (2) when $A$ is infinite.

Example 3.12. Let $A=\{1,2,3, \ldots\}$ and $N=\{1,2\}$. Let $E: P(N) \rightarrow P\left(P_{0}(A)\right)$ be given by: $E(1)=\{B \subseteq A|| A|B|<\infty\}$, and $E(2)=\{B \subseteq A \mid B$ is an infinite set $\}$. As the reader may check, $E^{*}=E$. Thus, $E^{*}(i)=E(i)$ for $i=1,2$, and (2) is satisfied. Nevertheless, the residual $\hat{E}$ is not stable: let $R^{i}, i=1,2$, be given by $a R^{i} b \Leftrightarrow a \geq b$ for all $a, b \in A$. Hence, by Lemma 3.1, $E$ has no Nash consistent representation. Observe that, indeed, condition (1) is violated here. For instance, for every natural number $\tau \geq 2$ the set $B_{\tau}:=\{1, \tau$, $\tau+1, \ldots\}$ is in $E^{*}(2)$, and hence $\cap\left\{B_{\tau} \mid \tau \geq 2\right\}=\{1\} \in I\left(E^{*}(2)\right)$. However, $A \backslash\{1\} \in$ $I\left(E^{*}(1)\right)$, so that $(1)$ is violated.

Remark 3.13. Let $|A|<\infty$ and let $E: P(N) \rightarrow P\left(P_{0}(A)\right)$ be a superadditive and monotonic effectivity function. If $n=|N|=2$, then, by (2), E has a Nash consistent representation if and only if $E$ is maximal, i.e. $E=E^{*}$. However, if $n \geq 3$, then $E$ may have an $N C$-representation without being maximal. Indeed, let $N=\{1,2,3\}$ and $A=\{a, b\}$. Consider the game form $\Gamma=\left(\{T, B\},\{L, R\},\left\{M_{1}, M_{2}\right\} ; \pi ; A\right)$, where $\pi$ is given by the following two matrices:

$$
M_{1}: \underset{B}{T}\left(\begin{array}{cc}
L & R \\
a & a \\
a & b
\end{array}\right) \quad M_{2}: T\left(\begin{array}{ll}
L & R \\
b & b \\
b & a
\end{array}\right)
$$

Then $\Gamma$ is Nash consistent. However, $E=E^{\Gamma}$ is not maximal: $E(3)=E(\{1,2\})=\{A\}$.

\section{Nash consistent representation of topological effectivity functions}

Throughout this section the set of alternatives $A$ is a topological space. The structure on $A$ is

$$
\mathscr{K}=\mathscr{K}(A)=\left\{B \in P_{0}(A) \mid B \text { is closed }\right\} .
$$

As before, let $N$ be the set of players. An effectivity function $E: P(N) \rightarrow P(\mathscr{K}(A))$ is called topological. A preference ordering $R$ on $A$ is continuous if for every $a \in A$ the sets $\{b \in A \mid a R b\}$ and $\{b \in A \mid b R a\}$ are closed. We denote by $V$ the set of all continuous preference orderings on $A$.

The main purpose of this section is to look for necessary and sufficient conditions for Nash consistent representation of topological effectivity functions. The following result 
provides a necessary condition for the case where $A$ is a normal space, i.e. for every pair of disjoint closed sets $B$ and $B^{\prime}$ there are disjoint open sets $U$ and $U^{\prime}$ with $B \subseteq U$ and $B^{\prime} \subseteq U^{\prime}$. The necessary condition is analogous to the one used in the finite case, namely (2).

Theorem 4.1. Let $A$ be a normal space and let the topological effectivity function $E$ have a Nash consistent representation on $V^{N}$. Then

$$
\left[B^{i} \in E^{*}(i) \text { for all } i \in N\right] \Rightarrow \bigcap_{i \in N} B^{i} \neq \emptyset .
$$

Proof. Let $\Gamma=\left(\Sigma^{1}, \ldots, \Sigma^{n} ; \pi ; A\right)$ be an NC representation of $E$ on $V^{N}$. Let $B^{i} \in E^{*}(i)$ for every $i \in N$. Assume, contrary to what we want to prove, that $\bigcap_{i \in N} B^{i}=\emptyset$. Then there exists a family of open neighbourhoods $U^{i}$ of $B^{i}$ with $\bigcap_{i \in N} U^{i}=\emptyset$. By Urysohn's Lemma there are continuous functions $f^{i}(i \in N)$ which are equal to 1 on $B^{i}$ and equal to 0 outside $U^{i}$. Consider the (continuous) preferences induced by the $f^{i}$ (also denoted by $f^{i}$ ) and let $\sigma$ be a Nash equilibrium in the associated game with outcome $x=\pi(\sigma)$. Since $\bigcap_{i \in N} U^{i}=\emptyset$, there exists $i \in N$ with $x \notin U^{i}$, i.e. $f^{i}(x)=0$. Since $\sigma$ is a Nash equilibrium, we have

$$
\begin{aligned}
& \left\{y \in A \mid \text { there is } \tau \in \Sigma^{i} \text { with } y=\pi\left(\tau, \sigma^{N \backslash\{i\}}\right)\right\} \\
& \subseteq L\left(x, f^{i}\right)=\left\{y \in A \mid f^{i}(y) \leq 0\right\} .
\end{aligned}
$$

This implies that $L\left(x, f^{i}\right) \in E(N \backslash\{i\})$, hence $B^{i} \cap L\left(x, f^{i}\right) \neq \emptyset$. Since $f^{i}$ is equal to 1 on $B^{i}$ and smaller than or equal to 0 on $L\left(x, f^{i}\right)$, we have a contradiction.

The converse of Theorem 4.1 is not true, as the following example shows.

Example 4.2. Let $A=[0,1]$, let $N=\{1,2,3\}$, and let $\lambda$ be the Lebesgue measure on $A$. Consider a topological EF on $A$ satisfying: $E(N)=\mathscr{K}(A) ; E(S)=\{B \in \mathscr{K}(A) \mid \lambda(B)>1 / 3\}$, if $|S|=2 ; E(i)=\{A\}$ for every $i \in N$; and $E(\emptyset)=\emptyset$. In this case, $E^{*}(i)=\{B \in \mathscr{K} \mid \lambda(B) \geq$ $2 / 3\}$ for every $i \in N$, and (4) is satisfied. Consider the following three functions defined on $A: u^{1}(x)=x, u^{2}(x)=1-x$, and $u^{3}(x)=\max (1 / 2-x, x-1 / 2)$. Define the profile $R^{N} \in V^{N}$ by

$$
x R^{i} y \Leftrightarrow u^{i}(x) \geq u^{i}(y) \text {, for all } x, y \in A \text { and all } i \in N .
$$

As may be verified, there is no point $a \in A$ such that $\lambda\left(L\left(a, R^{i}\right)\right)>1 / 3$ for $i=1,2,3$. See Fig. 1. Hence, Theorem A.1 implies that $E$ has no Nash consistent representation.

In order to formulate sufficient conditions for Nash consistent representation of a topological effectivity function, we introduce the following continuity notion. ${ }^{1}$ Here, a chain $\{B(\alpha) \mid \alpha \in I\}$ (where $I$ is an arbitrary index set) is a collection of subsets of $A$ with $B(\alpha) \subseteq B(\beta)$ or $B(\beta) \subseteq B(\alpha)$ for all $\alpha, \beta \in I$.

\footnotetext{
${ }^{1}$ Condition (5) is implied by the assumption that every $E(N \backslash\{i\})$ is closed in the upper topology. See Klein and Thompson (1984, Chap. 1).
} 


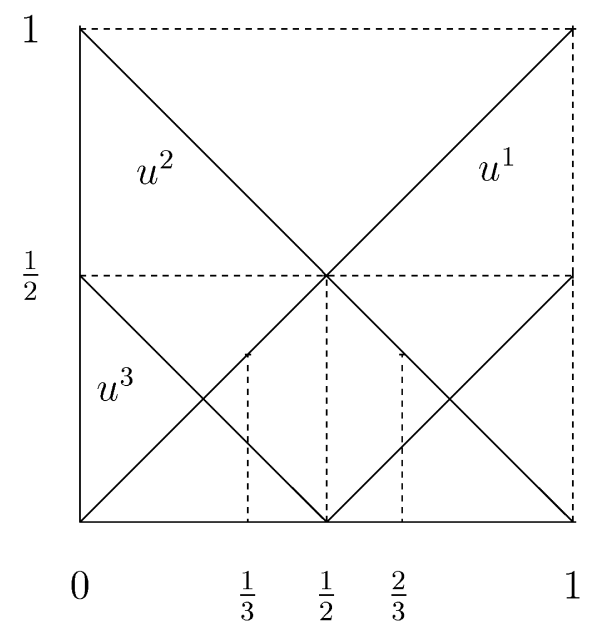

Fig. 1. The utility functions in Example 4.2.

For every chain $\{B(\alpha) \mid \alpha \in I\}$ :

$B(\alpha) \in E(N \backslash\{i\})$ for all $\alpha \in I$ implies $\bigcap_{\alpha \in I} B(\alpha) \in E(N \backslash\{i\})$.

Theorem 4.3. Let $A$ be a compact space and let $E: P(N) \rightarrow P(\mathscr{K}(A))$ be a superadditive and monotonic effectivity function satisfying (5). Assume that (4) is satisfied. Then $E$ has a Nash consistent representation on $V^{N}$.

Proof. We will show that $E$ satisfies condition (I.2) of Theorem A.1 (where $\mathscr{T}=\mathscr{K}(A)$ and $Q=V)$. Let $R^{N} \in V^{N}$. For every $i \in N$, denote by $B^{i}$ the set

$$
\left\{a \in A \mid L\left(a, R^{i}\right) \in E(N \backslash\{i\})\right\} .
$$

The sets $L\left(a, R^{i}\right)\left(a \in B^{i}\right)$ form a chain of closed subsets of $A$. By (5), the intersection $F^{i}$ of these sets is an element of $E(N \backslash\{i\})$. By continuity of the preference $R^{i}$ the compact set $F^{i}$ has a maximal (with respect to $R^{i}$ ) element $x^{i}$. Then $F^{i}=L\left(x^{i}, R^{i}\right)$ and $B^{i}=\left\{a \in A \mid a R^{i} x^{i}\right\}$.

We claim that $B^{i} \in E^{*}(i)$ for every $i \in N$. Since $B^{i}$ is a closed set, we only need to verify that $B^{i} \cap B^{\prime} \neq \emptyset$ for every $B^{\prime} \in E(N \backslash\{i\})$. Let $x^{\prime}$ be a maximal element of (the compact set) $B^{\prime}$ with respect to (the continuous preference) $R^{i}$. Then $B^{\prime} \subseteq L\left(x^{\prime}, R^{i}\right)$. By monotonicity of $E$, also $L\left(x^{\prime}, R^{i}\right) \in E(N \backslash\{i\})$. Hence $x^{\prime} \in B^{i}$ by definition of $B^{i}$. Since also $x^{\prime} \in B^{\prime}$ we have $B^{i} \cap B^{\prime} \neq \emptyset$ as required.

Since $B^{i} \in E^{*}(i)$ for every $i \in N$, (4) implies the existence of an $x \in \bigcap$ $\bigcap_{i \in N} B^{i}$. Thus, $L\left(x, R^{i}\right) \in E(N \backslash\{i\})$ for every $i \in N$, so that condition (I.2) of Theorem A.1 is satisfied.

Remark 4.4. Let the residual $\hat{E}: P(N) \rightarrow P(\mathscr{K}(A))$ be defined similarly as in Section 3: 


$$
\hat{E}(S)= \begin{cases}E^{*}(i), & \text { if } S=\{i\}, i \in N \\ \{A\}, & \text { if }|S|>1, S \subseteq N \\ \emptyset, & \text { if } S=\emptyset\end{cases}
$$

Suppose $A$ is a Hausdorff compact space (hence, normal) and suppose that $E$ satisfies (5). Firstly, if (4) is satisfied, then the element $x \in A$ as obtained in the proof of Theorem 4.3 is also in the core $C\left(\hat{E}, R^{N}\right)$; so $\hat{E}$ is stable. Secondly, let $\hat{E}$ be stable and suppose there are $B^{i} \in E^{*}(i)(i \in N)$ with empty intersection. Then we can construct preferences $f^{i}$ as in the proof of Theorem 4.1; since, for every $x \in A$ there is an $i$ with $x \notin B^{i}$ we have $C\left(\hat{E}, f^{N}\right)=\emptyset$, contradicting the stability of $\hat{E}$. Summarizing, for a Hausdorff compact space $A$ and assuming (5), stability of the residual $\hat{E}$ is equivalent to (4).

Remark 4.5. An effectivity function $E$ is called $(1, n-1)$-maximal if $E(i)=E^{*}(i)$ for every $i \in N$. Theorem 4.3 implies that a $(1, n-1)$-maximal, monotonic, superadditive topological effectivity function satisfying (5), has a Nash consistent representation if $A$ is compact. Cf. Moulin and Peleg (1982) and Keiding and Peleg (1999), where (stronger) maximality conditions are used to obtain representations that are strong Nash and coalition-proof Nash consistent, respectively.

Just as in Section 3, Corollary 3.2, further properties of the core of the residual $\hat{E}$ (see (6)) can be established. In particular, this core contains the Nash equilibrium outcomes of any Nash consistent representing game form, with equality for the game form $\Gamma_{0}$ constructed in the proof of Theorem A.1. So this last game form is maximal in the sense that it does not preclude any potential Nash equilibrium outcome. The following corollary, which coincides with Corollary 3.2 if the number of alternatives is finite, summarizes these observations.

Corollary 4.6. Let $E: P(N) \rightarrow P(\mathscr{K}(A))$ be an effectivity function. Then

(i) if $E$ has a Nash consistent representation $\Gamma$ with outcome function $\pi$, then $\pi(N E(\Gamma$, $\left.\left.R^{N}\right)\right) \subseteq C\left(\hat{E}, R^{N}\right)$ for all $R^{N} \in V^{N}$

(ii) if $A$ is compact and $E$ is superadditive, monotonic, satisfies (5), and $C\left(\hat{E}, R^{N}\right) \neq \emptyset$ for all $R^{N} \in V^{N}$, then $\pi\left(N E\left(\Gamma_{0}, R^{N}\right)\right)=C\left(\hat{E}, R^{N}\right)$ for all $R^{N} \in V^{N}$, where $\pi$ is the outcome function of $\Gamma_{0}$.

Proof. (i) Let $R^{N} \in V^{N}, \sigma \in N E\left(\Gamma, R^{N}\right)$, and $y=\pi(\sigma)$. Then $L\left(y, R^{i}\right) \in E^{\Gamma}(N \backslash\{i\})=$ $E(N \backslash\{i\})$ for every $i \in N$. Thus, if $i \in N$ and $B \in E^{*}(i)$, then $B \cap L\left(y, R^{i}\right) \neq \emptyset$. This implies that, for every $i \in N, y$ is not dominated via $\{i\}$ at $R^{N}$. Hence, $y \in C\left(\hat{E}, R^{N}\right)$. (ii) This follows from Remark 4.4 and the proofs of Theorems 4.3 and A.1.

A further, rather surprising, property of the core of the residual $\hat{E}$ is that it always contains a Pareto optimal outcome.

Theorem 4.7. Let the conditions of Theorem 4.3 be satisfied. Then for every $R^{N} \in V^{N}$ 


$$
C\left(\hat{E}, R^{N}\right) \cap P O\left(R^{N}\right) \neq \emptyset .
$$

Proof. Let $R^{N} \in V^{N}$. Let, for every $i \in N$, the point $x^{i}$ be as in the proof of Theorem 4.3. Then one easily verifies that

$$
C\left(\hat{E}, R^{N}\right)=\left\{x \in A \mid x R^{i} x^{i} \text { for all } i \in N\right\} .
$$

Since $A$ is compact and $C\left(\hat{E}, R^{N}\right)$ is nonempty and closed, this identity implies that $C(\hat{E}$, $R^{N}$ ) has an undominated element with respect to the (continuous) Pareto ordering which is also undominated in $A$, hence $C\left(\hat{E}, R^{N}\right) \cap P O\left(R^{N}\right) \neq \emptyset$.

An interesting implication of Theorem 4.7 and Corollary 4.6, part (ii), is that the game form $\Gamma_{0}$ constructed in the proof of Theorem A.1 admits a Pareto optimal Nash equilibrium for every profile of preferences. Note, furthermore, that Theorem 4.7 and this last remark hold, in particular, if the number of alternatives is finite (cf. Remark 3.8).

\section{Topological veto functions}

In this section the results of Section 4 will be applied to topological veto functions. In order to facilitate the general discussion, the case in which the set of alternatives is finite will be treated first. As before, $N$ is the set of players.

\subsection{The case $|A|<\infty$}

Let the set of alternatives $A$ be finite with $|A|=m \geq 2$. A veto function is a function $v$ : $P(N) \rightarrow\{-1,0, \ldots, m-1\}$ such that $v(\emptyset)=-1, v(S) \geq 0$ if $S \neq \emptyset$, and $v(N)=m-1$. The interpretation of the number $v(S)$ is that the coalition $S$ can veto any subset of alternatives with at most $v(S)$ elements. More formally, this is captured by defining an associated neutral (i.e. not depending on the names of the alternatives) effectivity function $E_{v}$ by

$$
E_{v}(S)=\left\{B \in P_{0}(A)|| A \backslash B \mid \leq v(S)\right\}=\left\{B \in P_{0}(A) \| B \mid \geq m-v(S)\right\}
$$

for every $S \in P(N)$. Conversely, as the reader may check, every neutral effectivity function is derived from some veto function. A veto function is monotonic if

$$
\left[S, S^{*} \in P(N), S \subseteq S^{*}\right] \Rightarrow v(S) \leq v\left(S^{*}\right),
$$

and it is superadditive if

$$
\left[S, S^{*} \in P(N), S \cap S^{*}=\emptyset\right] \Rightarrow v(S)+v\left(S^{*}\right) \leq v\left(S \cup S^{*}\right) .
$$

The interpretations of these properties are obvious: monotonicity means that larger coalitions have more power and superadditivity means that the union of disjoint coalitions has additional power compared to the coalitions operating separately. Clearly, a veto function is monotonic [superadditive] if, and only if, the associated effectivity 
function is monotonic [superadditive]. The existence of a Nash consistent representation for such an effectivity function can easily be characterized by applying Corollary 3.7.

Theorem 5.1. Let $A$ be a finite set of $m \geq 2$ alternatives, and let $v: P(N) \rightarrow\{-1,0, \ldots$, $m-1\}$ be a monotonic and superadditive veto function. Then the associated effectivity function $E_{v}$ has a Nash consistent representation if, and only if,

$$
\sum_{i \in N} v(N \backslash\{i\})>n(m-1)-m
$$

Proof. For every $i \in N$,

$$
E_{v}^{*}(i)=\left\{B \in P_{0}(A)|| B \mid \geq v(N \backslash\{i\})+1\right\} .
$$

Hence, (2) is satisfied if, and only if,

$$
\sum_{i \in N}(m-[v(N \backslash\{i\})+1])<m,
$$

which is (7).

\subsection{General topological veto functions}

Let $A$ be a compact metric space with metric $d$, let $\mathscr{B}$ be the $\sigma$-algebra of Borel sets of $A$, and let $\mu$ be a probability measure on $(A, \mathscr{B})$. A veto function is now a function $v$ : $P(N) \rightarrow[-1,1]$ with $v(\emptyset)=-1, v(N)=1$, and $v(S) \geq 0$ if $S \neq \emptyset$. The interpretation is similar as in the finite case: coalition $S$ can veto any subset of measure at most $v(S)$. Formally, the associated effectivity function $E_{v}$ will be restricted to closed sets:

$$
E_{v}(S)=\{B \in \mathscr{K}(A) \mid \mu(B) \geq 1-v(S)\} .
$$

Our approach here is motivated by Abdou (1988).

The definitions of monotonicity and superadditivity are identical to the earlier definitions for the finite case. Monotonicity of the veto function implies monotonicity of the associated effectivity function, as is easy to check. For superadditivity this implication does not hold, as the following examples show.

\section{Example 5.2.}

(a) Let $A=[0,1 / 2] \cup[1,3 / 2] ; \mu=\lambda$, where $\lambda$ is the Lebesgue measure; $N=\{1,2\}$; and let $v: P(N) \rightarrow[-1,1]$ be given by: $v(\emptyset)=-1 ; v(\{1\})=v(\{2\})=1 / 2$; and $v(N)=1$. Then $v$ is monotonic and superadditive. However, $E_{v}$ is not superadditive: $[0,1 / 2] \in E_{v}(\{1\}),[1,3 / 2] \in E_{v}(\{2\})$, and $[0,1 / 2] \cap[1,3 / 2]=\emptyset$.

(b) Let $A=[0,2] ; \mu(B)=\lambda(B \cap[0,1])$ for every Borel set $B$ of $A ; N=\{1,2\}$; $v(\emptyset)=-1 ; v(\{1\})=0$; and $v(\{2\})=v(N)=1$. Then $v$ is superadditive and monotonic. Now $[3 / 2,2] \in E_{v}(\{2\}),[0,1] \in E_{v}(\{1\})$, and $[0,1] \cap[3 / 2,2]=\emptyset$. Thus, again, $E_{v}$ is not superadditive. 
In Example 5.2(a), the set $A$ is not connected, and in Example 5.2(b), the support of $\mu, \operatorname{supp}(\mu)$, is not the whole set $A$. It turns out that, if these properties are avoided, then the associated effectivity function is superadditive if the veto function is.

Lemma 5.3. Let $A$ be connected and $A=\operatorname{supp}(\mu)$, and let $v$ be superadditive. Then $E_{v}$ is superadditive.

Proof. Let $S_{i} \in P_{0}(N), i=1,2, S_{1} \cap S_{2}=\emptyset$, and $B_{i} \in E_{v}\left(S_{i}\right), i=1,2$. Then $B_{i} \in \mathscr{K}(A)$ for $i=1,2$, and

$$
\mu\left(B_{1}\right) \geq 1-v\left(S_{1}\right), \mu\left(B_{2}\right) \geq 1-v\left(S_{2}\right) .
$$

Therefore,

$$
\begin{aligned}
\mu\left(B_{1}\right)+\mu\left(B_{2}\right) & =\mu\left(B_{1} \cap B_{2}\right)+\mu\left(B_{1} \cup B_{2}\right) \\
& \geq 2-v\left(S_{1}\right)-v\left(S_{2}\right) \geq 2-v\left(S_{1} \cup S_{2}\right) .
\end{aligned}
$$

Thus,

$$
\mu\left(B_{1} \cap B_{2}\right) \geq 1-v\left(S_{1} \cup S_{2}\right)+1-\mu\left(B_{1} \cup B_{2}\right) \geq 1-v\left(S_{1} \cup S_{2}\right) .
$$

Thus, $E_{v}$ is superadditive if we show that $B_{1} \cap B_{2} \neq \emptyset$. Suppose this is not the case, i.e. $B_{1} \cap B_{2}=\emptyset$. Then, by (7), $\mu\left(B_{1} \cup B_{2}\right)=1$. Hence, $B_{1} \cup B_{2}=A$ because $A=\operatorname{supp}(\mu)$. But this contradicts the connectedness of $A$.

The first main result in this section is the following theorem, which gives a sufficient condition for the effectivity function associated with a topological veto function to have a Nash consistent representation.

Theorem 5.4. Let $A$ be a compact and connected metric space and let $\mu$ be a probability measure on $(A, \mathscr{B})$ with $\operatorname{supp}(\mu)=A$. Let $v$ be a monotonic and superadditive veto function satisfying

$$
\sum_{i=1}^{n} v(N \backslash\{i\}) \geq n-1 .
$$

Then the associated effectivity function $E_{v}$ has a Nash consistent representation on $V^{N}$.

For the proof of this theorem we need two lemmas. Assume that the conditions in the theorem are fulfilled.

Lemma 5.5. Let $S \in P_{0}(N)$. Then

$$
E_{v}^{*}(S)=\{B \in \mathscr{K}(A) \mid \mu(B) \geq v(N \backslash S)\}
$$


Proof. First, suppose that $B \in \mathscr{\ell}(A)$ with $\mu(B)<v(N \backslash S)$. We show that $B \notin E_{v}^{*}(S)$. Define

$$
B_{t}=\left\{x \in A \mid d(x, B) \geq \frac{1}{t}\right\}, t=1,2, \ldots
$$

Then $B_{t} \in \mathscr{K}(A)$ for every $t=1,2, \ldots$, and $\cup_{t=1}^{\infty} B_{t}=A \backslash B$. Hence, there exists a $t_{0}$ with $\mu\left(B_{t_{0}}\right) \geq 1-v(N S)$. Thus, $B_{t_{0}} \in E_{v}(N \backslash S)$ and $B_{t_{0}} \cap B=\emptyset$. Therefore, $B \notin E_{v}^{*}(S)$.

Next, let $B \in \mathscr{K}$ satisfy $\mu(B) \geq v(N \backslash S)$. We assume $B \notin E_{v}^{*}(S)$ and derive a contradiction. By this assumption there exists $B^{\prime} \in E(N \backslash S)$ with $B^{\prime} \cap B=\emptyset$. As $\mu\left(B^{\prime}\right) \geq 1-$ $v(N \backslash S), \mu\left(B \cup B^{\prime}\right)=1$. Hence $B \cup B^{\prime}=A$, contradicting the connectedness of $A$.

For the next lemma, recall that $\mathscr{K}(A)$ with the Hausdorff metric $d_{H}$ is a compact metric space (Klein and Thompson, 1984, Chapter 4).

Lemma 5.6. $E_{v}(S)$ is closed in $\left(\mathscr{K}(A), d_{H}\right)$ for every $S \in P_{0}(N)$.

Proof. Let $\alpha \in[0,1]$. It is sufficient to prove that the set $\mathscr{K} *=\{B \in \mathscr{K}(A) \mid \mu(B) \geq \alpha\}$ is closed in $\left(\mathscr{K}(A), d_{H}\right)$. To show this, let $B(k) \in \mathscr{K}^{*}, k=1,2, \ldots$, and $B=\lim _{k \rightarrow \infty} B(k)$. For $B^{*} \subseteq A$ denote by $\chi_{B^{*}}$ the characteristic function of $B^{*}$, that is, $\chi_{B^{*}}(x)=1$ if $x \in B^{*}$ and $\chi_{B^{*}}(x)=0$ if $x \notin B^{*}$. Then, for $x \in B, \chi_{B}(x)=1 \geq \lim \sup _{k \rightarrow \infty} \chi_{B(k)}(x)$; and for $x \notin B, \chi_{B}(x)=0=\lim _{k \rightarrow \infty} \chi_{B(k)}(x)$. Hence,

$$
\begin{aligned}
\mu(B) & =\int_{A} \chi_{B}(x) \mathrm{d} \mu \geq \int_{A} \limsup _{k \rightarrow \infty} \chi_{B(k)}(x) \mathrm{d} \mu \\
& \geq \limsup _{k \rightarrow \infty} \int_{A} \chi_{B(k)}(x) \mathrm{d} \mu=\limsup _{k \rightarrow \infty} \mu(B(k)) \geq \alpha,
\end{aligned}
$$

where the second inequality follows from Fatou's Lemma. Thus, $B \in \mathscr{K}^{*}$.

We can now prove Theorem 5.4.

Proof of Theorem 5.4. The effectivity function $E_{v}$ is monotonic because $v$ is monotonic and superadditive by Lemma 5.3 because $v$ is superadditive. By Lemma 5.6, $E_{v}(N \backslash\{i\})$ is closed in $\left(\mathscr{K}(A), d_{H}\right)$ for every $i \in N$, so that in particular (5) is satisfied. In order to apply Theorem 4.3 we only have to prove

$$
\left[B^{i} \in E_{v}^{*}(i) \text { for all } i \in N\right] \Rightarrow \bigcap_{i=1}^{n} B^{i} \neq \emptyset
$$

Assume, on the contrary, that there exist $B^{i} \in E_{v}^{*}(i), i \in N$, such that $\bigcap_{i=1}^{n} B^{i}=\emptyset$. Let $D^{i}=A \backslash B^{i}, i \in N$. Then each $D^{i}$ is open, $\cup_{i=1}^{n} D^{i}=A$, and $\mu\left(D^{i}\right) \leq 1-v(N \backslash\{i\})$ for 
every $i \in N$ by Lemma 5.5. By (9), $\sum_{i=1}^{n} \mu\left(D^{i}\right) \leq 1$. Because $A=\operatorname{supp}(\mu)$, we must have $D^{i} \cap D^{j}=\emptyset$ for all $i \neq j$. Thus, $D^{1}, \ldots, D^{n}$ is a nontrivial open partition of $A$, contradicting the connectedness of $A$.

Condition (9) in Theorem 5.4 is far from necessary for the existence of a Nash consistent representation. Let, for instance, $A=[0,1]$ and let $\mu$ put weight $9 / 10$ on the one-point set $\{1\}$ and distribute weight $1 / 10$ uniformly over the interval. Consider a monotonic and superadditive veto function $v$ with $v(N \backslash\{i\})=8 / 10$ for every $i \in N$. Then, for $n>5$, (9) is not satisfied but

$$
E_{v}(N \backslash\{i\})=\{B \in \mathscr{K}(A) \mid 1 \in B\}=E_{v}^{*}(\{i\})
$$

for every $i \in N$, so that (4) is satisfied. Hence, Theorem 4.3 still implies that $E_{v}$ has a Nash consistent representation. In this example, the point 1 is an atom of $\mu$. For nonatomic probability measures, indeed, the next theorem shows that (9) is also a necessary condition.

Theorem 5.7. Let $A$ be a compact metric space and let $\mu$ be a nonatomic probability measure on $(A, \mathscr{B})$. Let $v$ be a monotonic and superadditive veto function. If the associated effectivity function $E_{v}$ has a Nash consistent representation on $V^{N}$, then (9) holds.

Proof. Assume, on the contrary, that

$$
\sum_{i \in N}[1-v(N \backslash\{i\})]>1
$$

Let $N_{0}=\{i \in N \mid v(N \backslash\{i\})<1\}$, hence $N_{0} \neq \emptyset$, and choose $\epsilon>0$ such that

$$
\sum_{i \in N}[1-v(N \backslash\{i\})]>1+n \epsilon, \text { where } \epsilon<\min \left\{1-v(N \backslash\{i\}) \mid i \in N_{0}\right\} .
$$

Next, choose $\hat{B}^{i} \in \mathscr{B}, i \in N$, as follows: if $i \notin N_{0}$, then $\hat{B}^{i}=A$; if $i \in N_{0}$, then choose $\hat{B}^{i}$ such that $\mu\left(\hat{B}^{i}\right)=v(N \backslash\{i\})+\epsilon$ and such that $\bigcap_{i \in N_{0}} \hat{B}^{i}=\emptyset$. These choices are possible because of nonatomicity of $\mu$ and (11). As $\mu$ is a probability measure on $(A, \mathscr{B})$, we can find closed sets $B^{i}, i \in N$, such that: (i) $B^{i}=A$ if $i \notin N_{0}$; (ii) $B^{i} \subseteq \hat{B}^{i}$ and $\mu\left(B^{i}\right)>v\left(N \backslash\{i\}\right.$ ), for $i \in N_{0}$ (see Dunford and Schwartz, 1988, p. 170).

Thus, we have obtained sets $B^{i} \in E_{v}^{*}(i), i \in N$, such that $\bigcap_{i \in N} B^{i}=\emptyset$. Hence, Theorem 4.1 (observing that a metric space is normal) implies that $E_{v}$ does not have a Nash consistent representation. Thus, we have a contradiction, which completes the proof.

We end this section with an application of Theorems 5.4 and 5.7. 
Example 5.8. In a city occupying an area of $1 \mathrm{~km}^{2}$ a public facility has to be located. Assume that there are three parties, $N=\{1,2,3\}$, and each majority of two parties $\{i, j\}$ can veto any area of at most $0 \leq v(\{i, j\}) \leq 1$. Hence, it is effective for any (closed) area of at least $1-v(\{i, j\}) \mathrm{km}^{2}$. Also, assume that $N$ is effective for any nonempty closed area. Thus, with $v(i)=0, i \in N$, so that single parties are only effective for the whole city, we obtain an effectivity function that is monotonic and superadditive. Theorems 5.4 and 5.7 imply that it has a Nash consistent representation if, and only if,

$$
v(\{1,2\})+v(\{1,3\})+v(\{2,3\}) \geq n-1=2 .
$$

\section{Concluding remarks}

In this paper the basic assumption was that constitutions can be modeled by effectivity functions, and the quest was for game forms (modeling the practical implementation of a constitution) that (i) represent the constitution, i.e. reflect the distribution of power implicit in the constitution; (ii) are stable in the sense of possessing at least one Nash equilibrium for any distribution of preferences. The main results are as follows. For general effectivity functions, i.e. without any special structure on the set of alternatives (social states), a necessary and sufficient condition for existence of a Nash consistent representation was given in terms of the polar effectivity for individuals: this condition amounts to the intuitive requirement that individuals should not be too powerful (condition (1)). A similar result was obtained for the case where the set of alternatives is a topological space and the effectivity function is topological (condition (4)). For the special case of topological veto functions (and with some additional requirements on the set of alternatives) we found as a necessary and sufficient condition for Nash consistent representation a simple numerical condition on the veto function (condition (9)). We would like to look upon these results as possibility results, witnessing the examples in Sections 3 and 5. Moreover, with respect to the Sen paradox we may note that among the Nash equilibria in the particular representation constructed in Appendix A, there are always Pareto optimal ones (cf. Remark 3.8, and Theorem 4.7 and the remark following it).

For the finite case, these results are related to those in Moulin and Peleg (1982), who consider strong Nash consistent representation and find stability and maximality of the effectivity function as necessary and sufficient conditions, and Keiding and Peleg (1999), who consider coalition-proof Nash consistent representation and find maximality of the effectivity function as a necessary and sufficient condition-given, of course, the other conditions of monotonicity and superadditivity.

\section{Acknowledgements}

The authors thank V.I. Danilov and two anonymous referees for useful remarks and suggestions. Section 4 in particular was substantially improved thanks to the comments 
of V.I. Danilov. Financial support from the Research School METEOR of the University of Maastricht, and from the Dutch Science Foundation NWO (grant no. B 46-417) is gratefully acknowledged.

\section{Appendix A. A necessary and sufficient condition for the existence of a Nash consistent representation}

Let $\mathscr{T}$ be a structure on the set of alternatives $A$, and let $Q \subseteq W$. The only further assumption that we make is that $Q$ is compatible with $\mathscr{T}$, that is, $L(a, R) \in \mathscr{T}$ for all $a \in A$ and $R \in Q$. As before, $N$ is the set of players.

Theorem A.1. Let $E: P(N) \rightarrow P(\mathscr{T})$ be an effectivity function. Then $E$ has a Nash consistent representation on $Q^{N}$ if, and only if, the following conditions are satisfied:

(I.1) $E$ is superadditive and monotonic.

(I.2) For every $R^{N} \in Q^{N}$ there exists an $x \in A$ such that $L\left(x, R^{i}\right) \in E(N \backslash\{i\})$ for all $i \in N$.

Proof. Necessity: let $\Gamma=\left(\Sigma^{1}, \ldots, \Sigma^{n} ; \pi ; A\right)$ be a Nash consistent representation of $E$. Because $E^{\Gamma}$ is superadditive and monotonic and $E(S)=E^{\Gamma}(S) \cap \mathscr{T}$ for every $S \in P_{0}(N)$, (I.1) holds. Let $R^{N} \in Q^{N}$. As $\Gamma$ is Nash consistent on $Q^{N}$, there exists an NE $\sigma$ of $(\Gamma$, $\left.R^{N}\right)$. Let $x=\pi(\sigma)$. Because $\sigma$ is a Nash equilibrium, $L\left(x, R^{i}\right) \in E^{\Gamma}(N \backslash\{i\})$ for every $i \in N$. Thus, $L\left(x, R^{i}\right) \in E^{\Gamma}(N \backslash\{i\}) \cap \mathscr{T}=E(N \backslash\{i\})$ for every $i \in N$.

Sufficiency: we shall first construct a representation of $E$ on $\mathscr{T}$. For $i \in N$ let $N^{i}=\{S \subseteq N \mid i \in S\}$ and denote

$$
M^{i}=\left\{m: N^{i} \rightarrow \mathscr{T} \mid m(S) \in E(S) \text { for all } S \in N^{i}\right\}
$$

A selection from $\mathscr{T}$ is a function $\phi: \mathscr{T} \rightarrow A$ such that $\phi(B) \in B$ for every $B \in \mathscr{T}$. Denote by $\Phi$ the set of all selections from $\mathscr{T}$. We now define a GF $\Gamma_{0}=\left(\Sigma^{1}, \ldots, \Sigma^{n} ; \pi\right.$; $A)$ as follows. The set of strategies of $i \in N$ is the set $\Sigma^{i}=M^{i} \times \Phi \times N$. Let $\sigma=$ $\left(\sigma^{1}, \ldots, \sigma^{n}\right) \in \Sigma^{1} \times \cdots \times \Sigma^{n}$, where $\sigma^{i}=\left(m^{i}, \phi^{i}, t^{i}\right)$ for $i \in N$. In order to define $\pi(\sigma)$ we introduce the following sequence of partitions of $N$. First, for $S \in P_{0}(N)$, we define an equivalence relation $\sim_{\sigma}$ on $S$ by

$$
i \sim{ }_{\sigma} j \Leftrightarrow m^{i}(S)=m^{j}(S)
$$

for all $i, j \in S$, and denote by $D(S)=D(S, \sigma)$ the partition of $S$ with respect to ${ }_{\sigma}$. Now let the first partition of $N$ be $H_{0}(\sigma)=\{N\}$. If $H_{k}(\sigma)=\left\{S_{k, 1}, \ldots, S_{k, l}\right\}$ is the $k$ th partition, where $k \geq 0$, then we define

$$
H_{k+1}(\sigma)=\bigcup_{j=1}^{l} D\left(S_{k, l}\right) .
$$


Clearly, there exists a minimal $r$ such that $H_{r}(\sigma)=H_{k}(\sigma)$ for all $k \geq r$. Write $H_{r}(\sigma)=$ $\left\{S_{1}, \ldots, S_{l}\right\}$. For each $1 \leq j \leq l$ there exists $B_{j} \in E\left(S_{j}\right)$ such that $m^{i}\left(S_{j}\right)=B_{j}$ for all $i \in S_{j}$. As $E$ is superadditive, $B=\bigcap_{j=1}^{l} B_{j} \neq \emptyset$. Moreover, $B \in \mathscr{T}$. Let $1 \leq i_{0} \leq n$ be the player with $i_{0} \equiv\left(t^{1}+\cdots+t^{n}\right) \bmod n$. Then we define $\pi(\sigma)=\phi^{i_{0}}(B)$.

We now prove that $\Gamma_{0}$ is a representation of $E$. Let $S \in P_{0}(N)$ and $B \in E(S)$. Choose $\sigma^{i}=\left(m^{i}, \phi^{i}, t^{i}\right)$ for every $i \in S$ such that

$$
m^{i}\left(S^{*}\right)=B \text { for all } S^{*} \supseteq S \text { and } i \in S \text {. }
$$

Then, by definition of $\pi, \pi\left(\sigma^{S}, \tau^{N \backslash S}\right) \in B$ for all $\tau^{N \backslash S} \in \Sigma^{N \backslash S}$. Hence $B \in E^{\Gamma_{0}}(S) \cap \mathscr{T}$, so $E(S) \subseteq E^{\Gamma_{0}}(S) \cap \mathscr{T}$.

In order to prove the converse inclusion let $C \in \mathscr{T} E(S)$. Then for every $B \in E(S)$, $B \backslash C \neq \emptyset$. Let further $\sigma^{S} \in \Sigma^{S}$ and $i_{0} \in N \backslash S$. (Recall that $E(N)=\mathscr{T}$, hence $S \neq N$.) Choose strategies $\tau^{i}=\left(m^{i}, \phi^{i}, t^{i}\right) \in \Sigma^{i}$ for every $i \in N S$ in the following way: $m^{i}(T)=A$ for all $T \in N^{i}$ and all $i \in N S$. Further, $\left[\sum_{i \in S} t^{i}+\sum_{i \in N \backslash S} t^{i}\right] \bmod n \equiv i_{0}$. Let $H_{r}\left(\sigma^{S}, \tau^{N \backslash S}\right)=\left\{S_{1}, \ldots, S_{l}\right\}$. Then $N \backslash S \subseteq S_{j}$ for some $1 \leq j \leq l$. Let $B_{h}=m^{i}\left(S_{h}\right)$ for $i \in S_{h}$ and $h=1, \ldots, l$. Then $B_{j}=A$. Hence, by superadditivity of $E, B:=\bigcap_{h=1}^{l} B_{h} \in E(S)$. Thus, $B \backslash C \neq \emptyset$. Now take $\phi^{i_{0}}(B) \in B \backslash C$. Then $\pi\left(\sigma^{S}, \tau^{N \backslash S}\right) \notin C$. Therefore, $C \notin E^{\Gamma_{0}}(S)$.

It remains to prove that $\Gamma_{0}$ is Nash consistent. Let $R^{N} \in Q^{N}$. By (I.2) there exists an $x \in A$ such that $L\left(x, R^{i}\right) \in E(N \backslash\{i\})$ for all $i \in N$. Choose $\sigma^{i}=\left(m^{i}, \phi^{i}, t^{i}\right) \in \Sigma^{i}$ for every $i \in N$ such that

(I.3) $m^{i}(N)=\{x\}$ for every $i \in N$.

(I.4) $m^{i}(N \backslash\{j\})=L\left(x, R^{j}\right)$ for all $i \in N \backslash\{j\}, j=1, \ldots, n$.

Clearly, $\pi(\sigma)=x$ and $\pi\left(\sigma^{N \backslash\{i\}}, \tau^{i}\right) \in L\left(x, R^{i}\right)$ for all $i \in N$ and $\tau^{i} \in \Sigma^{i}$. Thus, $\sigma$ is a Nash equilibrium.

\section{References}

Abdou, J., 1988. Neutral veto correspondence with a continuum of alternatives. International Journal of Game Theory 17, 135-164.

Abdou, J., 1995a. Nash and strongly consistent two-player game forms. International Journal of Game Theory $24,345-356$.

Abdou, J., 1995b. Solvability of two-player game forms with infinite sets of strategies. Mathematics of Operations Research 20, 213-226.

Arrow, K.J., 1967. Values and collective decision making. In: Laslett, P., Runciman, W.G. (Eds.), Philosophy, Politics, and Society, Third Series. Basil Blackwell, Oxford, pp. 215-232.

Danilov, V.I., Sotskov, A.I., 1991. Social Choice. Nauka, Moscow, in Russian.

Deb, R., Pattanaik, P.K., Razzolini, L., 1997. Game forms, rights, and the efficiency of social outcomes. Journal of Economic Theory 72, 74-95.

Dunford, N., Schwartz, J.T., 1988. Linear Operators, Part I: General Theory. Wiley Classic Library, New York. Dutta, B., 1984. Effectivity functions and acceptable game forms. Econometrica 52, 1151-1166.

Gaertner, W., Pattanaik, P.K., Suzumura, K., 1992. Individual rights revisited. Economica 59, 161-177. 
Gärdenfors, P., 1981. Rights, games, and social choice. Noûs 15, 341-356.

Gibbard, A., 1974. A Pareto-consistent libertarian claim. Journal of Economic Theory 7, 388-410.

Gurvich, V.A., 1976. Solvability of positional games in pure strategies. Zhurnal Vychislitel'noj Matematiki i Matematicheskoj Fiziki; Journal of Computational Mathematics and Mathematical Physics 15, 357-371.

Gurvich, V.A., 1989. Equilibrium in pure strategies. Soviet Math Doklady 38, 597-602.

Hurwicz, L., Schmeidler, D., 1978. Construction of outcome functions guaranteeing existence and Pareto optimality of Nash equilibria. Econometrica 46, 1447-1474.

Keiding, H., 1986. Stability of effectivity functions with an infinite set of alternatives. Methods of Operations Research 50, 519-530.

Keiding, H., Peleg, B., 1999. Representation of effectivity functions in coalition-proof Nash equilibrium: a complete characterization. Institute of Economics, University of Copenhagen, DP 99-21. Forthcoming in Social Choice and Welfare.

Klein, E., Thompson, A.C., 1984. Theory of Correspondences. Wiley, New York.

Moulin, H., Peleg, B., 1982. Cores of effectivity functions and implementation theory. Journal of Mathematical Economics 10, 115-145.

Peleg, B., 1998. Effectivity functions, game forms, games, and rights. Social Choice and Welfare 15, 67-80. Peleg, B., Winter, E., 2000. Constitutional implementation, Mimeo.

Sen, A.K., 1970. The impossibility of a Paretian liberal. Journal of Political Economy 78, 152-157.

Sugden, R., 1985. Liberty, preference, and choice. Economics and Philosophy 1, 185-205. 